\title{
Helmut Klages \\ In wiefern sind wir schon (oder noch) »Demokraten«?
}

Zusammenfassung: Ungeachtet aller Bemühungen um die Zustandsmessung der Demokratie und die Sorge um ihre zukünftige Entwicklung war die elementare Frage, inwieweit die einzelnen Mitglieder der Gesellschaft eigentlich "Demokraten" sind, erstaunlicher Weise kaum jemals aufgeworfen worden. Wendet man sich dieser Frage zu, dann gelangt man zu einem ambivalenten Ergebnis. Um dieses Ergebnis zu erzielen, muss man allerdings hinter die bekannten - für die Beantwortung der gestellten Frage aber keineswegs bedeutungslosen - Tatsachen eines absinkenden Parteienvertrauens, einer absinkenden Wahlbeteiligung, oder z.B. auch eines rückläufigen Stammwähleranteils zurückfragen und die elementare Tatsache ins Auge fassen, dass das Angebot einer Parteimitgliedschaft von der Bevölkerungsmehrheit von allem Anfang an nur spärlich angenommen wurde. Die »Parteiendemokratie« erscheint von daher einem problematischen Licht. Auch eine hohe »Demokratiezufriedenheit « und ein anwachsendes "politisches Interesse« vermögen über diesen Befund nicht hinwegzutäuschen. Dagegen verbirgt sich in dem - manchen Behauptungen zuwiderlaufenden - Fortbestehen der Anziehungskraft bestimmter Eigenschaften des politischen Spitzenpersonals eine bisher oft noch beiseitegeschobene Chance, die Systemzuwendung breiterer Bevölkerungsteile zu stärken.

Schlïsselwörter: Demokratie, Demokraten, Zivilkultur, Parteiendemokratie, Parteien

Helmut Klages, To what extent are we already (or still) "democrats"?

Summary: Irrespective of ongoing attempts at analyzing the quality of democracies and speculating about its future development, the elementary question, whether the majority of the individual members of society are 'democrats', has rarely been raised. Indeed, it is necessary, to look behind the well-known - although by no means unimportant - facts of a fading trust in political parties, of a growing unsteadiness of voting behavior, or a diminishing identification with political institutions, and to take into consideration the very elementary fact that the offer of acquiring membership in political parties was from the very beginning astonishingly seldom accepted by the majority of the population. Considering that fact, the whole context of 'party democracy' seems to slide. Even high levels of 'satisfaction with democracy' and 'interest in politics' are not appropriate to reconcile with this very fact. In contrast to that, the enduring attraction of certain personality traits of leading figures on the political stage offers - regardless of its frequent denial - a high potential of political mobilization and integration of the 'lonely crowd'.

Keywords: Democracy, Democrats, Civic Culture; Party Democracy, political parties 
Helmut Klages, Dr. rer. pol. und Dipl.-Volkswirt, Professor em. für empirische Sozialwissenschaften an der Deutschen Universität für Verwaltungswissenschaften Speyer.

Korrespondenzanschrift: klages@uni-speyer.de

\section{Die Ängste des Anfangs - und deren Überwindung}

Die Themenstellung, um die es gehen soll, ist ungewöhnlich. Zwar gibt es heute eine fast unübersehbare Literatur zur Demokratie als solcher, zu den politischen Parteien, oder auch zu den Wahlen und den dabei zum Tragen kommenden Wählermotiven, wie etwa auch zur Demokratiequalität in verschiedenen Ländern. Zu der Frage, inwiefern die Menschen im Lande »Demokraten« sind, findet sich aber kaum etwas. Gehen wir davon aus, dass bei der Beantwortung dieser Frage nach bestimmten politischen Einstellungen und Verbaltensweisen in der Bevölkerung zu fragen ist, dann mag sich die Politische Kulturforschung für zuständig erklären, aber auch sie hat sich bisher - ungeachtet langer Listen von Variablen abgefragter Einstellungen und Verhaltensweisen der Gesamtbevölkerung oder einzelner ihrer Teile - die Frage, um die es im vorstehenden Titel geht, bisher noch kaum direkt gestellt.

Wir wollen die naheliegende Frage, warum dies so ist, mit der Vermutung beantworten, dass eine bejahende Antwort auf die Ausgangsfrage dermaßen selbstverständlich zu sein scheint, dass es überflüssig anmuten mag, sie zum Gegenstand der Forschung zu erheben. Wir wollen uns durch diese Vermutung aber nicht entmutigen lassen, sondern uns vielmehr der Frage zuwenden, was über diejenigen politischen Einstellungen und Verhaltensweisen der Bevölkerung Deutschlands bekannt ist, die für die Beantwortung der Frage, inwiefern sie aus Demokraten besteht, relevant erscheinen. Wir wollen uns hierbei überwiegend - aber nicht ausschließlich - auf die »alte Bundesrepublik« bzw. Westdeutschland beschränken, da sich Ostdeutschland gegenwärtig immer mehr zu einem Sonderthema zu entwickeln scheint.

Wir stoßen, wenn wir uns dem empirischen Material zuwenden, sehr bald auf die Tatsache, dass es bezüglich der infrage kommenden Einstellungen und Verhaltensweisen eine Entwicklung gab, der die Dramatik nicht abgeht. Wie die Autoren einer 1959 im Rahmen der Internationalen Vergleichsstudie »The Civic Culture « ${ }^{1}$ durchgeführten Untersuchung feststellten, gab es in der damaligen Bundesrepublik ein »niedriges Niveau « der »Systemverbundenheit «, das sich bei Antworten auf Fragen herausstellte, die sich z.B. auf den »Stolz« auf die Regierung und sonstigen politischen Institutionen und auf die gefühlsmäßige Beziehung zu Wahlen, Wahlkämpfen und politischen Parteien bezogen. Es wurden weiter niedrige Niveaus der politikbezogenen Alltagskommunikation und der intensiveren Formen politischer Partizipation, so etwa der Parteimitgliedschaft und der Organisierung politischer Initiativen gefunden. Die befragten Deutschen offenbarten darüber hinaus aber auch Entfremdung, »Negativismus « und

1 Gabriel A.Almond \& Sidney Verba: The Civic Culture. Political Attitudes and Democracy in Five Nations, Princetown 1963, passim. 
Vertrauensmangel bezüglich politischer Parteien und weiterer struktureller Aspekte des politischen Systems.

Waren die damals befragten Deutschen also - von hierher gesehen - schon »Demokraten «?

Für einen beträchtlich großen Teil von Ihnen muss diese Frage offensichtlich rundweg verneint werden, denn $42 \%$ von ihnen antworteten auf die Frage, welche Zeit für Deutschland in diesem Jahrhundert »die beste gewesen sei, »das Dritte Reich«. Für die Autoren der betreffenden Studie ergab sich - auch in Verbindung hiermit - der Gesamteindruck, es müsse davon ausgegangen werden, dass nicht nur der gegenwärtige Zustand der Demokratie in Westdeutschland, sondern auch deren Zukunftsaussichten eher trübe aussähen.

Bei einer in den 70er Jahren durchgeführten Nachfolgestudie ${ }^{2}$ ergab sich allerdings ein völlig verändertes Bild, so dass deren Autoren folgerten, auf einen tiefgreifenden zwischenzeitlichen Wandel der »civic culture « in Westdeutschland seit den 50er Jahren schließen zu können. Sie legten hierbei u.a. die überraschende Entdeckung zugrunde, dass die Zahl derjenigen Befragten, die der Meinung waren, dass die jetzige Zeit für Deutschland die beste Zeit in diesem Jahrhundert sei, auf $81 \%$ angestiegen war, während der Anteil derjenigen, die das »Dritte Reich « nannten, auf nur mehr $5 \%$ gefallen war, d.h. gegen Null tendierte. Ebenso beeindruckend fanden sie es z.B., dass der Anteil derjenigen, die »stolz« auf die politischen Institutionen des Landes waren, im selben Zeitraum von $7 \%$ auf $31 \%$, der Anteil derjenigen, die der Demokratie den Rang der bestmöglichen Regierungsform zuerkannten, von $74 \%$ auf $90 \%$ und der Anteil der politisch Interessierten von $27 \%$ auf $50 \%$ angestiegen war.

Die Autoren der Nachfolgestudie meinten, aus derartigen Ergebnissen die Folgerung ableiten zu können, die Demokratie habe in Deutschland ihren Einzug gehalten und dieses Urteil schloss ganz offensichtlich die Auffassung ein, die Deutschen seien nunmehr - jedenfalls zum überwiegenden Teil - »Demokraten« geworden.

\section{Die überraschende Karriere der »normativen « Einstellungen}

In der Tat ergibt sich bei einem Gesamtrückblick auf die Anlaufzeit der damaligen Bundesrepublik der Eindruck, dass nach einer Periode der nachwirkenden Betäubung durch das Kriegsende und des mit ihm einhergehenden totalen Systemzusammenbruchs zumindest einige Indikatoren, die als einschlägig gelten können, eine erstaunlich eindeutige Hinwendung der ganz überwiegenden Bevölkerungsmehrheit zu einer »demokratischen « Grundeinstellung erkennen lassen. Es handelt sich hierbei um die Idee der Demokratie als solche, wie auch um deren grundlegende Merkmale wie die Abhaltung von allgemeinen, gleichen, freien und geheimen Wahlen, die Gewährleistung von Meinungsfreiheit, den auf einem Parteienpluralismus aufbauenden Parlamen-

2 Gabriel A. Almond \& Sidney Verba eds., The Civic Culture Revisited, Newbury Park et al. 1989. Bei den nachfolgend wiedergegebenen Ergebnissen wurde in erster Linie das Kapitel »Changing German Political Culture« von David P. Conradt, p. 212 ff., zugrunde gelegt. 
tarismus, das Recht auf Opposition, wie auch die Möglichkeit einer »Parteienregierung « ${ }^{3}$. Unter Zugrundelegung dieser Kriterien ließe sich in der Tat feststellen, dass den (West-)Deutschen schon relativ frühzeitig mit vollem Recht die Qualifikation, »Demokraten« zu sein, zuzusprechen war.

Genau betrachtet handelte es sich dabei allerdings nur um einen ganz bestimmten wenn auch grundlegend wichtigen - Ausschnitt aus der Gesamtheit der in Frage kommenden Merkmale (oder »Indikatoren«).

Konkret gesagt ging es um einen speziellen Komplex von Einstellungsmerkmalen, den man in der politischen Kulturforschung im allgemeinen als »normativ « bezeichnet, während die öfters als "performativ « bezeichneten Einstellungsmerkmale, bei denen es sich um die Beurteilung des tatsächlichen Funktionierens der Demokratie handelt, jedenfalls teilweise schon frühzeitig sehr deutlich nachhinkten und von einem bestimmten Zeitpunkt an eine Tendenz zum weiteren Absinken entwickelten.

So sank z. B. das »Vertrauen in die politischen Parteien und die Politiker «on einem mittleren Wert, den es noch in den 70er Jahren aufwies, in den nachfolgenden Jahren zunehmend auf einen inzwischen erreichten beinahe unglaublichen Tiefstand von wenigen Prozentpunkten ab. Die inzwischen gängig gewordene Rede von der Politikerund Parteien-»Verdrossenheit « der Bevölkerung findet hier scheinbar eine Bestätigung, die über jeden Zweifel erhaben zu sein scheint. ${ }^{4}$

\section{Das Nachbinken und nachfolgende Absinken der "performativen "Einstellungen}

Ohne diesbezüglich in allzu viele Details einzutreten, wollen wir registrieren, dass es für ein rückläufiges Wählervertranen in die Parteien in der Umfrageforschung zahlreiche Belege gibt, so z.B. die Antworten auf die direkt gestellte Frage nach dem Parteienvertrauen, die sich mit den Antworten auf das Vertrauen in andere Institutionenbereiche vergleichen lassen. Die Parteien befinden sich hier seit geraumer Zeit regelmäßig auf einem der allerletzten Rangplätze. Allerdings muss den Kritikern dieses Ergebnisses Recht gegeben werden, wenn sie darauf hinweisen, dass sich die gewöhnlich pauschal gestellte Frage nach dem Vertrauen in »die « Parteien auch auf alle diejenigen Parteien bezieht, welche die Antwortenden nicht gewählt haben, gegen die sie sich also entschieden haben und denen sie verständlicher Weise misstrauisch gegenüberstehen mögen. ${ }^{5}$

3 Vgl. Dietrich Thränhardt: Demokratie in Deutschland, bpb.de/nachschlagen/Lexika/handwoerterbuch-politisches System/202006/ Demokratie in Deutschland Demokratie in Deutschland.

4 Vgl. etwa Everhard Holtmann, Der Parteienstaat in Deutschland. Erklärungen, Entwicklungen Erscheinungsbilder, 2. überarbeitete und erweiterte Auflage, Bonn 2017, S. 175 ff. Für die an dieser Stelle, wie auch für eine größere Zahl nachfolgend genannter Ergebnisse empirischer Forschung detaillierte Belege anzugeben und zu dokumentieren erübrigt sich insoweit, als diese in allgemein zugänglichen Quellen auffindbar sind.

5 Vgl. hierzu nochmals Everhard Holtmann, Der Parteienstaat in Deutschland, a.a.O., S. 177, unter Bezugnahme auf den Wahlforscher Bernhard Weßels. 
Ähnlich verhält es sich mit den teils schockierend negativen Antworten auf diverse Fragen nach der Einstellung zu »den « Politikern, denen gegenüber ebenfalls der Einwand sticht, dass hier überall auch alle diejenigen Politiker mit abgefragt werden, denen die Wähler aus unterschiedlichen Gründen negativ gegenüberstehen, so dass die überwiegend vorhandenen positiven Einzelbewertungen in den pauschal formulieren Fragen und Antworten nicht angemessen zur Geltung kommen.

Den auf die Parteien bezogenen Fragebeantwortungen stehen außerdem die Ergebnisse der auf einzelne Parteien bezogenen Fragen nach der sogenannten »Parteiidentifikation « entgegen, deren Beantwortungen zwar allesamt sehr deutlich rückläufige persönliche Bindungen der Bevölkerungsmehrheit an die Parteien erkennen lassen, so dass von einer »Identifikation « im vollen Sinne des Wortes in der Tat nur noch im Ausnahmefall gesprochen werden kann. Die verfügbaren Daten lassen aber immerhin bei größeren Teilen der Wählerschaft noch vorhandene - wenngleich in einem zunehmenden Maße wechselnde - Sympathien gegenüber bestimmten Parteien erkennen, die vom Gesamttrend her gesehen sicherlich abklingen, deren Vorhandensein aber zur Kenntnis genommen werden muss. Wie schwach diese allerdings bereits geworden sind, lässt sich z.B. daran erkennen, dass die sogenannten »Wechselwäbler inzwischen die sogenannten »Stammwäbler zahlenmäßig überrundet haben, zugleich aber auch daran, dass die Zahl der sog. »unentschlossenen « Wähler, die bis kurz vor dem Wahltermin schwanken, welcher Partei sie ihre Stimme geben sollen, von Bundestagswahl zu Bundestagswahl anstieg, um endlich Im Wahljahr 2017 die $40 \%$-Marke, d.h. also einen erstaunlich hohen Stand zu erreichen. ${ }^{6}$

\section{Der Blick auf die "performativen" Einstellungen bedarf der Ergänzung}

Zusammenfassend gesagt dürfte ungeachtet der zahlreichen Einwände, die berechtigter Weise einem Teil der Daten entgegenzubringen sind, Anlass dazu bestehen, von einem sehr beträchtlichen Vertrauens- und Bindungsverlust der Parteien zu sprechen, wie auch von einem Fraglichwerden ihrer Repräsentationsfunktion, d.h. ihrer Fähigkeit, die Wünsche, Interessen und Bedürfnisse der Bevölkerung in die Zentren des politischen Entscheidens zu vermitteln.

Es lässt sich allerdings darüber streiten, ob die bloße Beschaffenheit der Performanz-Merkmale tatsächlich ausreichend viel darüber aussagt, ob Menschen die Eigenschaft »Demokrat « zu sein, zugesprochen werden kann oder nicht. Die Unzufriedenheit mit den gegebenen Umständen kann ja - um nur zwei diametral verschiedenartige Möglichkeiten zu nennen - einerseits auf eine mangelhafte Erfüllung realistischer und berechtigter Erwartungen aufgrund von Qualifikationsmängeln, Fehlhandlungen, oder Versäumnissen des Führungspersonals, oder auch auf ökonomische Krisenzustände zurückzuführen sein, deren Eintritt unbeeinflussbaren Umständen zuzuschreiben ist. Sie kann andererseits aber auch auf die Enttäuschung unerfüllbarer, weil utopischer Er-

6 Vgl. zu näheren Angaben etwa Manfred G. Schmidt, Das politische System Deutschlands, 3.aktualisierte Aufl., München 2007, S, 75 f. 
wartungen auf Seiten der Bevölkerung zurückgehen, die möglicherweise von anonymen »Influencern «, oder auch von den »negativistisch « eingestellten Redaktionen konventioneller Medien angeheizt worden sein mögen.

Die »demokratische« Qualifikation wird man der Bevölkerung nur im letzteren Fall wegen einer verantwortungslosen Überforderung der politischen Führung absprechen wollen, die man - um ein gegenwärtig in Umlauf befindliches Wort zu gebrauchen ohne weiteres als »populistisch« bezeichnen kann. Die diesbezügliche Urteilsbildung muss letztlich von der genaueren Prüfung der jeweiligen Umstände abhängig gemacht werden, wobei ganz offensichtlich ein sehr anspruchsvoller, moralische und kognitive Qualifikationen auf allen Seiten berücksichtigender Beurteilungsstandard zugrunde gelegt werden muss, in den auch der Einfluss dritter Kräfte und Mächte einzurechnen ist.

\section{Demokraten obne Parteimitgliedschaft?}

Mit den bisher behandelten beiden Gruppen von Einstellungsmerkmalen ist aber keineswegs bereits der ins Auge zu fassende Gesamtbefund erfasst. Was bisher noch völlig außer Acht gelassen ist, ist vielmehr die Frage nach dem politischen Verhalten der (West-)Deutschen, der naturgemäß bei der Beantwortung der Frage nach der Stärke und Ausprägung ihrer »demokratischen « Eigenschaften neben - oder sogar noch vor den Einstellungsmerkmalen eine ausschlaggebende Bedeutung zukommt.

Wir wollen an dieser Stelle E. Wiesendahl zu Wort kommen lassen, dessen Standpunkt sich zwar außerhalb des politikwissenschaftlichen Mainstreams bewegt, dem unseres Erachtens aber eine ernsthafte Diskussionswürdigkeit und grundsätzliche Anerkennung nicht abgesprochen werden kann. ${ }^{7}$

Wir können hierbei unmittelbar an die provozierende Feststellung dieses Autors anknüpfen, dass nicht etwa nur die Demokratie in Deutschland, sondern die liberale Demokratie im allgemeinen ein »elitäres Repräsentationsverständnis" habe, denn sie billige »dem Volk« nicht mehr als die letztlich passive Rolle »des vertrauenden Wählers« $\mathrm{zu}$, die er unmittelbar nach einer vollzogenen Wahl einzunehmen habe. Im Prinzip werde er nach dem Wahlakt, in welchem er seine Souveränität unwiderruflich abgebe, »auf die Zuschauerbühne des politischen Geschehens « verwiesen und aus der laufenden politischen Entscheidungsfindung ausgeschaltet.

Dieser Gewaltakt werde allerdings, so schränkt er ein, durch die Existenz politischer Parteien erheblich abgemildert. Diese würden den an den Rand des politischen Raums gedrängten Menschen eine reale Partizipationschance - und gleichzeitig auch eine politische Heimat - anbieten. Einzig und allein diese Tatsache versöhne das Konzept der liberalen Demokratie mit dem Volkssouveränitätsprinzip, zu dem sie sich ja eigentlich grundsätzlich bekenne.

7 Vgl. hierzu und im folgenden Elmar Wiesendahl, Partizipation und Engagementbereitschaft. In Parteien, Manuskript im Rahmen des Reports »Demokratie in Deutschland 2011 « der Friedrich-Ebert-Stiftung, o.J. 
Wiesendahl hält im Anschluss an diese kritische Feststellung aber gleich noch eine zweite Ernüchterung bereit, indem er feststellt, dass es in Deutschland (wie auch in anderen Ländern) »trotz der hohen Einflusspotenziale der Parteimitgliedschaft ... nur eine kleine Minderheit der Bevölkerung « gebe, »die von dieser Form der politischen Partizipation Gebrauch macht«. Wiesendahl nennt unter Bezugnahme auf die Freiwilligensurveys 1999 und 2004 eine Zahl von »weniger als $5 \%$ « der Befragten, die bekunden, Parteimitglieder zu sein. ${ }^{8}$

Wir können hinzufügen, dass es in der Tat in der Bundesrepublik unter Einbeziehung aller jeweils existierenden Parteien niemals mehr als insgesamt ca. 2,4 Millionen Parteimitglieder gegeben hat. Wiesendahl führt seine Analyse aber erbarmungslos fort, indem er feststellt, dass es unter den relativ wenigen Mitgliedern »ein krasses Partizipationsgefälle« gebe. Bei der Hälfte der Mitglieder herrsche völlige Apathie, so dass von realer Beteiligung keine Rede sein könne. Die andere Hälfte teile sich in »Dauer- und Gelegenheitsaktive auf, wobei ein Kern aus 10 - $20 \%$ Daueraktiven ... das Binnenleben der Parteien aufrecht erhält.«9

Erschwerend kommt, wie sich ergänzen lässt, aber noch hinzu, dass die genannte Zahl von 2,4 Millionen Parteimitgliedern eine Maximalzahl ist, die im Jahr 1976 erreicht wurde und seitdem - nach einer bis ca. 1990 anhaltenden Phase der Stagnation insbesondere aufgrund eines fortwährenden Mitgliederverlust der beiden damals noch großen Volksparteien, darüber hinaus aber auch aufgrund von Verlusten der kleineren Parteien mit Ausnahme der Grünen - stark zusammenschrumpfte.

\section{Die ausgebliebene Parteimitgliedschaft der Bevölkerungsmebrheit als Handicap}

Obwohl die Mitgliedschaft in einer Partei nur eines derjenigen Verhaltensmerkmale ist, die für die Beantwortung unserer Leitfrage Bedeutung haben, wollen wir uns bereits an dieser frühen Stelle eine Reflexionspause gönnen.

Im Grunde genommen vermitteln uns schon die vorstehend mitgeteilten Tatsachen die das erwartbare Endergebnis der Untersuchung an wichtigen Punkten vorwegnehmende Erkenntnis, dass die mehrheitliche Ausschlagung des Angebots der Parteimitgliedschaft und damit einer die engen Partizipationsbegrenzungen der repräsentativen Demokratie relativierenden institutionalisierten Beteiligungschance auf gravierende Begrenzungen der in der Bevölkerung vorhandenen Demokratieorientierung schließen lässt. ${ }^{10}$

8 Manfred G. Schmidt, a.a.O., S. 100, gibt an, dass im Jahr 2010 »nur noch 1,9\% der beitragsberechtigten Bevölkerung - deren Alter je nach Parteizugehörigkeit variierte- einer der sechs wichtigsten deutschen Parteien « angehörten.

9 Vgl. zu näheren Angaben unter detaillierter Bezugnahme auf Ergebnisse empirischer Forschung E. Holtmann, a.a.O., S. $229 \mathrm{ff}$.

10 Wir lassen hier das gesamte Faktenmaterial der Bürgerbeteiligung auf kommunaler Ebene und in den Bundesländern außer Acht, bei dessen Einbeziehung sich nur relativ wenige Änderungen ergeben, weil die Kerngruppe der aktiven Träger der Bürgerbeteiligung überwiegend aus bereits vielfach aktiven und politisch vernetzten Bürgern und großenteils aus Par- 
Es wird mit dieser Feststellung bewusst eine Abweichung von der in der politikwissenschaftlichen Literatur bis heute vorherrschenden Interpretation des geringen Ausmaßes der Parteimitgliedschaft in Kauf genommen. Zwar wird dieses allenthalben als Faktum registriert und als Defizit bewertet. Es wird hierbei aber in der Regel nur die Rückläufigkeit der Parteimitgliedschaft seit ca. 1990 zur Kenntnis genommen und behandelt, wobei jedoch der eigentliche Grundsachverhalt ausgeblendet wird, dass Parteimitgliedschaft in der Bundesrepublik von allem Anfang an ein Ausnabmesachverbalt war. Es lässt sich beim Blick auf die Zahlen nicht verheimlichen, dass die Bevölkerungsmehrheit von der Chance der Partizipation durch Parteimitgliedschaft nie Gebrauch machte, d.h. aber die mit dem Repräsentationsprinzip verbundene definitive Ausschließung von der dauerhaften Wahrnehmung der eigenen Souveränitätsrechte relativ widerstandslos hinnahm.

Dieser ebenso fundamentale wie auch im vollen Sinne des Wortes fragwürdige Sachverhalt wird dadurch nicht verharmlost, dass er sich, wie sich aufgrund der Ergebnisse internationaler Vergleiche feststellen lässt, auch in einer Mehrzahl anderer Länder findet. Aber auch die Tatsache, dass es aufgrund des Ergebnisses einschlägiger Studien in Deutschland permanent ein nennenswertes Potenzial von Befragten gab, die angaben, den Eintritt in eine Partei bereits zu irgendeinem Zeitpunkt »in Erwägung gezogen « zu haben, verharmlost diesen Sachverhalt keineswegs. Diese Tatsache verschärft und akzentuiert ihn vielmehr, denn sie signalisiert ja, dass es offenbar nur bei einer politisch besonders interessierten Minderheit der Bevölkerung überhaupt einen diesbezüglichen Entscheidungsprozess gab, der allerdings peinvoller Weise in der ganz überwiegenden Mehrzahl der Fälle zu Ungunsten des Parteibeitritts verlief.

\section{Gründe für die ausgebliebene Parteimitgliedschaft der Bevölkerungsmebrheit}

Natürlich muss sich, wenn man erst einmal die Tatsache eines stark reduzierten Starts der Parteiendemokratie an einem strategisch wichtigen Punkt zur Kenntnis genommen und intellektuell bewältigt hat, die Frage nach dem »Warum « erheben.

Dass die Chance einer Parteimitgliedschaft anfangs nur sehr verhalten angenommen wurde, wird man weitgehend der Wirkung des »Ohne mich«-Prinzips in der unmittelbaren Nachkriegszeit ${ }^{11}$ zuschreiben können, das nicht nur die Fortexistenz des Nazismus in der Bevölkerung verhinderte, sondern zunächst auch dem Interesse an der akti-

teimitgliedern besteht, so dass sie keine substanzielle Erweiterung der Gruppe der politischen Aktivbürger mit sich bringt. Vgl. hierzu die Ergebnisse der Untersuchung der gegenseitigen Beziehungen zwischen Partizipationsformen auf verschiedenen Ebenen bei Oscar W.Gabriel und Norbert Kersting, Politisches Engagement in deutschen Kommunen: Strukturen und Wirkungen auf die politischen Einstellungen von Bürgerschaft, Politik und Verwaltung, in: Bertelsmann Stiftung, Innenministerium Baden-Württemberg (Hrsg.), Partizipation im Wandel, 2014, S. 43 ff.).Die Autoren gelangen u.a. zu der Feststellung, dass »die bisher erfolgte Erweiterung der Beteiligungsmöglichkeiten« in erster Linie » den bereits aktiven Teilen der Bürgerschaft zusätzliche Einflussmöglichkeiten auf die Politik gegeben « hat (S. 47).

11 Vgl. hierzu Helmut Schelsky, Die skeptische Generation. Eine Soziologie der deutschen Jugend, Düsseldorf/Köln 1957, passim. 
ven Wahrnehmung demokratischer Partizipationschancen deutliche Begrenzungen auferlegte. Bezüglich der Erklärung des zeitlich begrenzten Mitgliederaufschwungs bei den beiden großen Volksparteien seit dem Ende der 60er Jabre wird man Wiesendahl zustimmen können, der auf die antagonistisch verlaufende Mobilisierungswirkung der damals hart umkämpften Grundentscheidung für oder gegen eine maßgebliche Verstärkung der gesellschaftsinternen Demokratisierung, wie auch für oder gegen eine massive Aufrüstung im kalten Krieg unter Einbeziehung der Atomenergie hinweist. Für die Erklärung der massiven nachfolgenden Rückläufigkeit der Parteizugehörigkeit gibt Wiesendahl - unter Zusammenfassung der diesbezüglichen Diskussion - ein eng geschnürtes Bündel verschiedenartiger Gründe an, das wir zunächst in unveränderter Form wiedergeben wollen. Im Spiele waren ihm zufolge »Faktoren wie Anreizschwächen der Parteien, Individualisierung und Milieuerosion, Wertewandel und kognitive Mobilisierung, Entpolitisierung und Medien-/Freizeitgesellschaft, sowie Vertrauenskrise zwischen Parteien und Bürgerschaft.«

Gönnen wir uns für die Erfassung der besonders maßgeblichen Faktoren etwas mehr Zeit, dann haben wir uns vor Augen zu führen, dass der gesellschaftliche Modernisierungsprozess in ersten Anfängen schon seit dem Ende des 19.Jh., mit zunehmender Wucht aber seit der zweiten Hälfte des 20.Jh. zur Auflösung derjenigen durch schroffe Trennlinien (oder »cleavages«) voneinander abgegrenzten »sozialmoralischen Milieus «12 führte, die seit dem Beginn der Industrialisierung den entstehenden politischen Parteien eine gewissermaßen »natürliche« gesellschaftliche Grundlage geboten hatten. Mit einer gewissen idealtypischen Zuspitzung ausgedrückt entwickelte sich anstelle dessen nunmehr eine kleinteilige soziale Differenzierung, in welcher - in der Bevölkerungsmehrheit - individuell gestaltete Lebensvollzüge und -entwürfe unter Nutzung einer immer unübersehbarer werdenden Zahl von Optionschancen eine zunehmende Bedeutung erhielten. Die programmatische Positionierung der politischen Parteien wurde unter diesen Bedingungen insofern zum Problem, als ihnen zunehmend ihre »angestammten« Klientele abhandenkamen. Insbesondere eine Partei, die nach wie vor "große Partei« sein wollte, stand nunmehr der Zwangslage gegenüber, als sogenannte »Volkspartei« für möglichst Alle attraktiv zu ein, sich dabei aber der Konkurrenz anderer Parteien ausgesetzt zu sehen, die genau dasselbe wollten - oder vielmehr wollen mussten. ${ }^{13} \mathrm{Da}$ man sich angesichts dessen »in der Sache « kaum mehr eindeutig unterscheiden konnte, nahm der entstehende Parteienwettbewerb zwangsläufig die Form einer Überbietungskonkurrenz an - es sei denn, dass eine Partei umschwenkte und einer anderen die unredliche Bedienung unerfüllbarer Träume vorwarf, wobei sie sich aber der Gefahr aussetzen musste, als »reaktionär« oder zumindest als »unsozial« gebrandmarkt zu werden. So oder so galt es, nach der Wahl in Koalitionsgespräche

12 Vgl. zur Ersteinführung dieses Begriffs Mario Rainer Lepsius: Parteiensystem und Sozialstruktur. Zum Problem der Demokratisierung der deutschen Gesellschaft, in: Gerhard Ritter (Hrsg.), Deutsche Parteien vor 1918, Köln 1973, S. 68.

13 Vgl. hierzu - auf dem Hintergrund zahlreicher vorangehender Veröffentlichungen, in denen dieses Thema abgehandelt wird - Helmut Klages: Absturz der Parteiendemokratie?, Frankfurt am Main, 2018, passim. 
einzutreten, die nunmehr allerdings unter dem Zwang standen, realistisch zu sein, d.h. der Knappheit der Mittel gerecht zu werden und ggf. von vorherigen Versprechungen zurückzutreten, d.h. also die Wähler enttäuschen zu müssen, damit aber letztlich auch die Axt an die Wurzeln des Wählervertrauens zu legen.

\section{Das zunebmende Einflussgewicht des politischen Spitzenpersonals als Erklärungsfaktor}

Wiesendahl ist Recht zu geben, wenn er auf die Überlagerung dieses Vorgangs durch einen zweiten hinweist. Er spricht in diesem Zusammenhang von der Entwicklung eines »kanzlerdemokratischen" oder »demoautoritären« Politikstils seit Helmut Schmidt. Es wird damit die Tatsache eines weitgehenden Austrocknens der örtlichen oder ortsnahen Basisorganisationen der Parteien und ihrer zunehmenden Bedeutungslosigkeit in Verbindung mit einem stark angewachsenen Einflussgewicht des politischen Spitzenpersonals angesprochen, das im Fall der Regierungsbeteiligung einer Partei in der Regel zugleich Regierungsämter ausübt und im Zentrum der Medienaufmerksamkeit steht und dessen faktische Dominanz in allen wichtigen Entscheidungsfragen alles schulbuchartige Reden von »innerparteilicher Demokratie« in ein problematisches Licht taucht..

Natürlich steht dieser Vorgang in kausalem Zusammenhang mit dem Absterben der früheren gesellschaftlichen Bodenhaftung der Parteien und der wachsenden Bedeutung ihrer Agilität im Wettrennen um zugkräftige Themen im Kampf um die Wählerstimmen, bei welchem der Zwang zu einer permanenten Abstimmung mit einer regional ausdifferenzierten Basis ein enormes Hindernis wäre. Er steht aber auch in sehr engen Zusammenhang mit einer weitgehenden Übernahme der mit einem anwachsenden Aufgabenvolumen des Staates umfangreicher und komplexer werdenden vorbereitenden Arbeiten zur Gesetzgebung und der damit zusammenhängenden Abstimmung mit den organisierten gesellschaftlichen Interessen durch die der Regierung zuarbeitenden ministeriellen Apparate. Diese Entwicklung relativiert nicht nur die Bedeutsamkeit der Tätigkeit der Parteien, sondern lässt darüber hinaus selbst noch die Parlamente bis hin zum Bundestag einschließlich der parlamentarischen Ausschüsse ungeachtet ihrer zentralen Ansiedelung in einem zunehmenden Maße zu quasi-notariellen Legitimierungsgremien degenerieren.

Alle diese miteinander verkoppelten Vorgänge finden ihren logischen Abschluss in einem fortschreitenden Macht- und Einflussgewinn der Bundesregierung, sodass sich nicht nur von einem Gestalt-und Funktionswandel der »Parteiendemokratie« sprechen lässt, die sich vielmehr unmerklich in einen historischen, d.h. der Vergangenheit zuzurechnenden Systemtyp verwandelt. 


\section{Der wachsende Abstand zwischen beabsichtigter und faktischer Wablbeteiligung als Problem}

Bevor wir bezüglich unserer Leitfrage zu abschließenden Ergebnissen gelangen, haben wir uns zunächst noch mit der Kernfrage der Wahrnehmung des Wablrechts, zu befassen, das öfters als eine entscheidende Grenzmarke der Demokratie gegenüber allen Varianten nicht- oder pseudodemokratischer Verhältnisse angesehen wird.

Auf den ersten Blick betrachtet scheinen wir hier zur Einnahme einer entspannteren Haltung berechtigt zu sein, denn jedenfalls bei der Frage nach der Absicht der Wahlbeteiligung, die sich zwischen den Bundestagswahlterminen auf den jeweils bevorstehenden Wahltermin bezieht, gaben während der gesamten Laufzeit des »Politbarometers «, d.h. also von den 70er Jahren des vorigen Jahrhunderts bis heute, jeweils $80-90 \%$ der Wahlberechtigten an, dass sie die Absicht hatten, zur Wahl zu gehen, wobei sie auch überwiegend - allerdings zunächst noch ohne definitive Festlegung - eine bestimmte Partei im Auge hatten.

Eine eindrucksvollere Demonstration des Demokratiebekenntnisses scheint man sich kaum wünschen zu können. Die Parteiendemokratie, der vorstehend eine Blessur nach der anderen zugefügt werden musste, scheint hier eine überraschende, zur Einschränkung kritischer Vorbehalte zwingende Vitalität zu demonstrieren.

Dass ein Freudenausbruch an dieser Stelle sehr verfrüht wäre, erweist sich jedoch sobald man nur weiter in die Daten eindringt. Fragt man nämlich nach der faktischen Wablbeteiligung, dann stößt man auf eine insgesamt gesehen sehr enttäuschende absinkende Entwicklung, die sich entgegen den vorher bestehenden Beteiligungsabsichten insbesondere bei den Kommunal- und Landtagswahlen feststellen lässt, während sich bei den Bundestagswahlen ein schwankende Beteiligung mit schwächerer, aber immerhin noch deutlich erkennbarer rückläufiger Trendrichtung abzeichnet..

Unter Absehung von Einzelheiten lässt sich feststellen, dass die faktische Wahlbeteiligung - unabhängig von der Stärke der hier erkennbar werdenden Abweichung - im Ganzen gesehen sehr deutlich hinter der vorangegangenen Bekundung der Wahlabsicht zurückblieb.

Aus den einschlägigen Fakten lässt sich darüber hinaus aber auch ablesen, dass die innerhalb der Bevölkerungsmehrheit vorherrschende, wie auch durch den Druck der öffentlichen Meinung massiv unterstützte Absicht der Wahlbeteiligung in Wirklichkeit kein »echter " Demokratie-Indikator, sondern vielmehr ein Indiz der völlig andersartigen Tatsache ist, dass das Wählen innerhalb der Bevölkerungsmehrheit den Status eines Kriteriums woblanständigen Verhaltens errungen hat. Man ist bereit zu wählen, so wie man in früheren Zeiten in der Regel dazu bereit war, sonntags in die Kirche zu gehen nicht unbedingt um einem religiösen Bedürfnis Genüge zu tun, sondern sehr häufig deshalb, weil »man« das so machen musste, wenn man den Eindruck vermeiden wollte, ein gesellschaftlicher Außenseiter und kein ordentlicher Bürger zu sein. Diese Deutung wird - empirisch gesehen - sehr nachdrücklich durch die Tatsache unterstützt, dass die Wahlbereitschaft mit keinem der zahlreichen anderen zur Verfügung stehenden politi- 
schen Einstellungs- oder Verhaltensindikatoren in einer signifikanten statistischen Beziehung steht.

Man könnte zugespitzt sagen, dass diese Bereitschaft eher ein Indikator einer amorphen Konformismusneigung als der Internalisierung eines demokratischen Imperativs ist. So betrachtet braucht es nicht zu verwundern, wenn im Einzelfall mit zunehmender Annäherung an den Wahltermin die Gründe für einen Verzicht auf die faktische Wahlbeteiligung zunehmen und durchsetzungskräftiger werden - handle es sich nun um das allzu schöne oder allzu schlechte Wetter, um das eigene körperliche Befinden und die damit ggf. verbundene Unabkömmlichkeit, um die Entdeckung, dass man mit der Partei, die man im Auge hat, doch nicht eng genug übereinstimmt, um ihr die eigene Stimme zu geben, oder auch um eine unüberwindliche Unfähigkeit, zwischen den verschiedenen sich anbietenden, allesamt nur begrenzt attraktiven Parteien zu entscheiden, deren Einfluss auf das was am Ende geschieht, ohnehin fragwürdig ist.

\section{Wer zur Wabl geht, muss deshalb nicht schon "Demokrat" sein}

Geht man davon aus, dass die Wahlentscheidung - soweit sie überhaupt stattfindet - in sehr vielen Fällen nur noch von einer relativ schwachen und möglicherweise zeitlich begrenzten Verbundenheit mit der gewählten Partei getragen wird und vielfach auf hilfsweise herangezogenen Motiven wie einer evtl. noch vorhandenen familiären Tradition, oder der mehr oder weniger zufälligen Entdeckung eines attraktiv anmutenden Einzelangebots im Wahlkampf beruht, dann wird man Mühe haben, ihr bezüglich der Beantwortung unserer Leitfrage nach dem Demokratiestatus der Bevölkerungsmehrheit einen hohen Stellenwert einzuräumen. Wer zur Wahl geht, muss, mit anderen Worten, nicht deshalb bereits ein »Demokrat« sein. Allgemeiner ausgedrückt würde man wahrscheinlich »Äpfel mit Birnen« verwechseln, wenn man alle heutigen politischen Systeme, in denen Wahlen stattfinden, an denen sich möglicherweise sogar die große Mehrheit der Bevölkerung beteiligt, unterschiedslos als »Demokratien « bezeichnen wollte. Es führt kein Weg daran vorbei, an jede Wahl die Anforderung zu richten, dass sie sowohl allgemein, unmittelbar und frei, wie auch gleich und geheim sein muss, um als »demokratisch« gelten zu können.

Von zumindest gleicher Bedeutung ist aber auch, dass die Wähler den Wahlakt auf dem Hintergrund einer eindeutigen, klar reflektierten, von persönlicher Bejahung und Übereinstimmung getragenen und gegen temporäre Störungen und Versuchungen immunen Entscheidung vollziehen. Nur hinzugehen und pflichtgemäß »ein Kreuzchen« zu machen, kann nicht bereits bedeuten, einen »demokratischen« Wahlakt zu vollziehen, sondern stellt u.U. nur seine Karikatur dar! 


\section{Hohe »Demokratiezufriedenheit «nd großes "politisches Interesse " sind nicht gleichbedeutend mit hoher Demokratieorientierung}

Als sehr überraschend müssen bei alledem nun allerdings zwei bisher noch nicht angesprochene Ergebnisse der Demokratieforschung angesehen werden, denen man - jedenfalls bei flüchtigem Hinsehen - genügend Gewicht zuschreiben könnte, um unsere bisherigen eher skeptisch stimmenden Ergebnisse am Ende doch noch ins Positive zu wenden.

Das eine ist die Tatsache, dass die "Demokratiezufriedenheit - mit Daten des »Politbarometers « gemessen - in (West-)Deutschland im Anschluss an die Anlaufperiode nach 1948 über Jahrzehnte hinweg mit nur kurzen Unterbrechungen relativ hoch gewesen ist, d.h. über dem Durchschnittswert der Europäischen Union lag und in den letzten 10 - 15 Jahren sogar eine weitere Steigerung erlebte, die sich, nebenbei bemerkt, auch in Ostdeutschland beobachten ließ.

Das andere ist die ebenso überraschende Tatsache, dass das "politische Interesse" mit demselben Erhebungsinstrument gemessen - in der Bevölkerung Deutschlands in den zurückliegenden Jahrzehnten eher zugenommen hat. Wie dir verfügbaren Daten erkennen lassen, bewegte sich diese Einstellungsgröße etwa bis zum Ende der 90er Jahre relativ konstant auf einem mittleren Niveau, um anschließend langsam aber stetig anzusteigen, wobei als besonders bedeutsam gelten kann, dass sich dieser Anstieg vor allem bei jungen und jüngeren Menschen unter 30 abzeichnete.

Beide Entwicklungen können allerdings nicht als Ausdruck einer stabilen bzw. sogar ansteigenden Hinwendung der Bevölkerungsmehrheit zur Demokratie gedeutet werden. Im Fall der »Demokratiezufriedenheit « verbietet sich dies bereits angesichts der Tatsache, dass diejenige Einflussgröße, die in erster Linie die relativ stabile Höhenlage dieser Variable erklärt, gar nicht im Bereich des politischen Systems, sondern vielmehr im wirtschaftlichen Bereich zu suchen ist. Wie der Blick auf die Daten erweist, folgt jedenfalls die mit diesem Instrument gemessene Entwicklung der »Demokratiezufriedenheit« relativ genau der Entwicklung der Variable »Eigene Wirtschaftslage«, wobei zu beachten ist, dass die auf die Demokratiezufriedenheit bezogene Fragevorgabe im Politbarometer - ungeachtet einiger Formulierungsänderungen im Laufe der Zeit stets relativ offen gestellt worden war, d.h. in keiner Weise auf das politische System im engeren Sinn des Wortes zielte, sodass sie ohne weiteres das Einfließen nichtpolitischer Assoziationen in die Beantwortung zuließ. ${ }^{14}$

14 Die ausschlaggebende Bedeutung, welche der individuellen Wirtschaftslage bei der Bestimmung der Höhe der mit Hilfe des «Politbarometers" gemessenen Demokratiezufriedenheit zukommt, lässt sich z.B. auch aus dem Ergebnis einer von M. Tausendpfund durchgeführten Regressionsanalyse der Demokratiezufriedenheit ablesen, in welche eine größere Zahl faktischer oder möglicher Einflussgrößen einbezogen worden war. Vgl. hierzu Markus Tausendpfund, Zufriedenheit mit der Demokratie. Ein Blick auf die Einstellungen der wablberechtigten Bevölkerung von 1977 bis 2016, in: ISI-Informationsdienst sozialer Indikatoren, Ausgabe 60, S. $29-34$. 
Den Anstieg des »politischen Interesses « in der Bevölkerungsmehrheit und insbesondere bei ihren jüngeren Teilen mit einer Attraktivitätssteigerung der deutschen Demokratie erklären zu wollen, verbietet sich deswegen, weil hierdurch ein massiver inhaltlicher Interpretationsbruch und -widerspruch erzeugt würde. Eher stellt sich in diesem Zusammenhang die Erinnerung an die nachdenkliche Schlussfrage im Deutschlandkapitel der Studie »Civic Culture Revisited « (vgl. oben) ein, ob die Institutionen der nur wenige partizipative Möglichkeiten zur Verfügung stellenden deutschen Demokratie den Bedürfnissen der ihrer demokratischen Kindheit entwachsenden Deutschen auf Dauer angemessen sein würden. Anders ausgedrückt ist zu fragen, ob das zunehmende politische Interesse vielleicht in erster Linie etwas mit einer auf dem Hintergrund vergleichbarer früherer Erregungswellen ansteigenden Protestneigung zu tun hat, die sich aktuell mit Themenstellungen verbindet, die von der Politik faktisch oder angeblich vernachlässigt werden, so z.B. mit einem »von Menschen gemachten « Klimawandel, mit der »Altersarmut«, mit einer sich noch immer steigernden »ungerechten Einkommens- und Vermögensverteilung « und der Existenz zunehmend zahlreicher »abgehängter « Menschen, wie letztlich aber auch mit einer - noch immer als Möglichkeit in Betracht zu ziehenden - »ungesteuerten Massenzuwanderung « von Menschen, die zum großen Teil ein »better life« im Blick haben und Deutschland mit einem paradiesischen Garten Eden mit unbegrenzten Möglichkeiten verwechseln.

Müsste diese Frage bejaht werden, wofür Vieles spricht, dann würde dies zwar - vgl. auch weiter oben - nicht eo ipso ein Grund dafür sein, den Deutschen die demokratische Qualität absprechen zu wollen. Es wäre aber mit Sicherheit ein Grund für die Annahme, dass die Bedingungen für die Förderung starker demokratischer Einstellungen und Verbaltensweisen der Bevölkerungsmehrheit in Deutschland, d. h. also nicht nur der kleinerer Minderheiten, noch nicht in ausreichendem Maße gewäbrleistet sind und dass deren Demokratiequalität und -niveau u.a. auch deshalb zu wünschen übrig lässt.

Um kein Missverständnis aufkommen zu lassen sei nochmals ausdrücklich darauf hingewiesen, dass im vorliegenden Zusammenhang nicht von Ostdeutschland, sondern bevorzugt von Westdeutschland die Rede ist.

\section{Schlussfolgerungen}

Der»Wablzyklus" der Regierungsbewertungen als Erschwernis des "Demokrat-Seins"

In die Schlussfolgerungen, die aus der vorliegenden Analyse zu ziehen sind, sind wir mit der vorstehenden kritischen Feststellung eigentlich bereits eingetreten. Eine erste Zielsetzung dieses abschließenden Kapitels soll zunächst sein, den Belegen für die Richtigkeit und Notwendigkeit dieser Feststellung noch einen starken Akzent hinzuzufügen.

Die Grundlage hierfür soll eine Datenanalyse und -interpretation der Bewertung der deutschen Bundeskanzler von 1977 bis 2018 liefern. ${ }^{15}$

15 Vgl. hierzu die Grafik in Helmut Klages, Absturz der Parteiendemokratie?, S. 134.

ZfP 67. Jg. 3/2020 
Beim Blick auf die Daten fallen zwei Dinge sofort ins Auge: Zum einen die extreme Schwankungsbreite der Bewertungen, die von den höchsten Skalenpunkten des verwendeten Messinstruments bis in dessen Negativbereich unterhalb der Skalenmitte reicht; zum anderen aber auch die Tatsache, dass sich sehr deutlich eine Rhythmik dieser Bewertungsschwankungen abzeichnet, die darin besteht, dass sich - jedenfalls bei den Bundeskanzlern Helmut Schmidt, Helmut Kohl und Gerhard Schröder - die positiven Bewertungsspitzen nach vorausgegangenen Bewertungsanstiegen in unmittelbarer Nähe der Bundestagswahlen finden, nach denen die Bewertungen mehr oder weniger kontinuierlich bis zu den jeweiligen Tiefstpunkten absinken, um anschließend einem neuen Zyklus Platz zu machen, der demselben Muster folgt, d.h. sein Zentrum bei der jeweils nachfolgenden Bundestagswahl hat.

Die Daten enthüllen mit dieser Rhythmik eine Quasi-Gesetzlichkeit des politischen Lebens der Bundesrepublik, die in Ansätzen bereits vor mehreren Jahren entdeckt und mit einer gewissen Unterkühlung als »Wahlzyklus« bezeichnet wurde. ${ }^{16}$ Der Datenverlauf, der diesen Vorgang über mehr als 40 Jahre hinweg verfolgbar werden lässt, macht allerdings auch deutlich, dass er eine wesentlich dramatischere Bezeichnung verdient. Geht man von der bereits weiter oben angesprochenen Tatsache eines weitgehenden Austrocknens der örtlichen oder ortsnahen Basisorganisationen der Parteien und ihrer zunehmenden Bedeutungslosigkeit in Verbindung mit einem stark angewachsenen Einflussgewicht des politischen Spitzenbereichs aus, dann wird deutlich, dass sich dieser Systemwandel - denn darum handelt es sich hierbei - mit der Entstehung einer Turbulenz verbindet, welche das Regieren, wie auch das »Demokrat-Sein « gleichermaßen sehr erschweren muss. Indem nämlich die Wünsche, Erwartungen und Forderungen der Bevölkerungsmehrheit und deren Beantwortung mit Versprechungen, die von den Parteien im Bundestagswahlkampf im Rahmen einer am Wünschbaren orientierten Überbietungskonkurrenz gemacht werden, viel direkter als früher auf dem Kabinettstisch und insbesondere auch bei dem/der Inhaber/in der Kanzlerrolle landen, geraten die Inhaber der Spitzenämter viel stärker als früher in die schwierige Situation, nach dem Wahlkampf dessen Folgen unter Orientierung am Machbaren »abarbeiten « zu müssen, d.h. neben Erwartungserfüllungen auch Erwartungsversagungen nach außen hin vertreten zu müssen.

Die in den Daten erkennbar werdende Bewertungsturbulenz, die den Inhaber/innen der Kanzlerrolle zuteil wird, ist somit das Ergebnis einer Situation, die sich dadurch auszeichnet, dass sich der Machtzuwachs, welcher der Spitze zukommt, mit einer vor allem bei ihr selbst aufschlagenden Zentralisierung des gesellschaftlichen Erwartungsdrucks verbindet, der in früheren Zeiten im Fall seiner Nichterfüllung zum guten Teil von den Parteien, die ihn hervorgerufen hatten, im Wege einer disziplinierenden Füh-

16 Siehe hierzu Jürgen W.Falter und Hans Rattinger, Die deutschen Parteien im Urteil der öffentlichen Meinung von 1977-1994, in: Oscar W.Gabriel/Oskar Niedermayer/Richard Stöss (Hrsg.), Parteiendemokratie in Deutschland. Rabmenbedingungen - Entwicklungen - Perspektiven, Opladen, S. 495 - 513; vgl. weiter Jürgen Maier, Politische Skandale - Quelle der Politikverdrossenheit?, in: Heribert Schatz/Patrick Rössler/Jörg-Uwe Nieland (Hg.), Politische Akteure in der Mediendemokratie, Wiesbaden 2002, S. 223-241. 
rung der jeweiligen Gefolgschaften auch selbst wieder aufgefangen werden konnte. Indem dieser Aspekt der vormaligen »Repräsentations «-Leistung der Parteien zusammen mit anderen ihrer Bestandteile inzwischen weitgehend entfällt, gerät die regierende Spitze unter den ungefilterten Druck von Enttäuschungsfolgen, den sich ihre von verschiedenen Parteien her koalierenden Mitglieder gegenseitig als »schwarzen Peter « zuschieben, was jedoch ihre Fähigkeit zu einem kraftvollen und einheitlichen Regierungshandeln schwächt oder im Grenzfall unmöglich macht.

Der »Frust«, der gegenwärtig die »Groko« begleitet, demonstriert diese Problematik sehr anschaulich. Da es um ihre eigene Wiederwahl geht, verstrickt sich die Spitze letztlich selbst in den Handlungszwang der Erwartungserzeugung, mit der sie fataler Weise aber gleichzeitig ihre eigene Belastung weiter vermehrt und ihren Handlungsspielraum weiter verringert. Für die Bürger wird die Situation zunehmend intransparent, wie auch - unter Medieneinfluss - intolerabel. Während ihre »Demokratiezufriedenheit« bei gesicherter ökonomischer Prosperität nur wenig in Mitleidenschaft gezogen werden mag, sinkt ihr Vertrauen in die Politik. Ihre Demokratieorientierung schlafft weiter ab und enthält in einem zunehmenden Maße protestbereite Züge. ${ }^{17}$

\section{Die Sonderstellung Angela Merkels als Kanzlerin; ibre Bedeutung für die Demokratieorientierung der Bevölkerungsmehrheit}

Ein genauerer Blick auf die Daten vermittelt allerdings die Erkenntnis, dass A. Merkel als Kanzlerin von der Problematik ihrer Vorgänger viel weniger betroffen war. Zwar erlebte sie in den zurückliegenden Jahren ebenfalls einige Einbrüche ihrer Beliebtheit Diese waren jedoch erstens nur im Fall der Bundestagswahl von 2009 an den von ihren Vorgängern her gewohnten »Wahlzyklus« gekoppelt, während sie die nachfolgende Wahl von 2013 singulärer Weise ohne jegliche "Delle« überstand. Zweitens erreichten die vorangegangenen Einbrüche bei weitem nicht die Tiefpunkte ihrer Vorgänger, sondern bewegten sich allesamt deutlich über der Null-Linie, d.h. also - wenn auch abgeschwächt - im positiven Bereich. Selbst die »Flüchtlingskrise« von 2015, die ihr sehr weitgehend persönlich zugerechnet wurde, die eine größere Teile der Bevölkerung erfassende Angst- und Wutwelle hervorrief und die den Aufstieg der AfD zu einer numerisch bedeutenden Partei nach sich zog, vermochte zwar ihre Beliebtheit zu reduzieren, jedoch nicht ernsthaft zu gefährden, geschweige denn zu zerstören, so dass sie sich anschließend wieder sehr weitgehend regenerieren konnte.

Es erscheint von daher unausweichlich, A. Merkel eine Sonderstellung zumindest gegenüber den vorstehend genannten Kanzlern zuzubilligen. Diese Sonderstellung wird auch durch weitere Sachverhalte bestätigt, so z.B. durch mehr oder weniger deutliche Erhöhung der Zufriedenheitswerte zahlreicher Politikbereiche bei denjenigen Teilen der Bevölkerung, die A. Merkel eine maßgebliche persönliche Einwirkung auf sie zurechneten, wie z.B. auch durch die Tatsache, dass sie bei der Mehrheit der Wähler fast aller politischen Parteien gute Bewertungen erhielt, so dass ihre Beliebtheitsbasis

17 Vgl. hierzu auch Renate Köcher in der FAZ vom 20.11.2019, S. 10.

ZfP 67. Jg. 3/2020 
keineswegs an »ihre eigene« Partei gebunden, sondern relativ unabhängig von der jeweiligen Parteisympathie in der gesamten Bevölkerungsmehrheit verankert war. Es kann darüber hinaus auch kaum einen Zweifel daran geben, dass die relativ gute Bewertung, die der Bundesregierung über den größeren Teil der letzten Jahre hinweg zuteil wurde, der herausragenden personenbezogenen Bewertung von A. Merkel zuzuschreiben ist. ${ }^{18}$

Nutzung der potenziellen Attraktivität des politischen Spitzenpersonals für die Steigerung der Demokratieorientierung der Bevölkerung als Handlungsperspektive

Werfen wir an dieser Stelle einen Blick zurück, dann können wir zunächst feststellen, dass wir hinsichtlich unserer Leitfrage nach dem Demokratieniveau der deutschen Mehrheitsbevölkerung insgesamt gesehen zu einem »ambivalenten « Ergebnis gelangt sind, was angesichts der Gegebenheiten auch kaum anders zu erwarten war. Gleichzeitig haben wir aber auch Erkenntnisse über eine Reihe von Einflüssen auf das Demokratieniveau der Bevölkerungsmehrheit gewonnen, was insofern ein Gewinn ist, als sich damit der Blick auf verschiedenartige Möglichkeiten der zukünftigen Entwicklung und deren Beeinflussbarkeit verbinden kann.

Greifen wir, um bei dem letzteren Punkt anzusetzen, unter Außerachtlassung mehrerer anderer Möglichkeiten nur zwei hauptsächliche Alternativen eines möglichen Tätigwerdens heraus, so gelangt mit einer gewissen Unvermeidlichkeit zunächst die Frage nach einer möglichen Korrektur der inzwischen schon historisch gewordenen Abstinenz der Bevölkerungsmehrheit bezüglich der Parteimitgliedschaft ins Blickfeld.

Obwohl einer solchen Korrektur eine vorrangige Dringlichkeit zugesprochen werden mag, lässt sich von ihr allerdings kaum ein befriedigendes Ergebnis erwarten. Den Parteien ist zuzubilligen, dass sie die sich diesbezüglich anbietenden Möglichkeiten allesamt längst durchgespielt haben - und auf »Granit gebissen « haben. Die Perspektiven einer die Parteimitgliedschaft der Mehrheit der Bevölkerung anvisierenden Vitalisierung der Demokratie scheinen - abgesehen von einzelnen Neugründungen unter Nutzung der Gunst des Augenblicks - ausgeschöpft zu sein. Als Ursache lässt sich angeben, dass sich in der Bevölkerungsmehrheit inzwischen die Erkenntnis durchgesetzt hat, dass bezüglich der Parteien allenfalls eine kurzfristige Zustimmungsreaktion auf attraktive Angebote im Vorfeld von Wahlentscheidungen, d.h. also nicht etwa eine Dauermitgliedschaft ohne unmittelbar absehbarem Nutzwerteffekt, rational ist.

Unsere Analyse hat demgegenüber allerdings auch zu der überraschenden Erkenntnis hingeführt, dass - in Verbindung mit Wahlentscheidungen, darüber hinaus aber auch mit einer hochgradigen Chance des wahlunabhängigen Verbleibens - die Anziebungskraft personaler Eigenschaftskombinationen - oder sagen wir kurz: von Persönlichkeiten - zu denjenigen gesellschaftlichen Grundsachverhalten rechnet, denen selbst ein noch so radikaler Wandel der Verhältnisse »nichts anzuhaben« vermag. Mit dieser einen Erkenntnis lässt sich aber zwanglos die weitere verbinden, dass das Nachdenken 
über die »Vitalisierung der Demokratie« bisher weitgehend an der Chancenhaltigkeit der hier sichtbar werdenden Durchsetzungskraft eines gesellschaftlichen Grundsachverhalts vorübergegangen ist, d.h. dessen politisches Potenzial übersehen, oder sogar bewusst ausgeklammert hat.

Setzt man demgegenüber genau an dieser Stelle an, dann kann man aus den Beobachtungen, die sich bezüglich der Besonderheiten der gesellschaftlichen Zuwendung zu der Kanzlerin Angela Merkel feststellen lassen, grundsätzliche Folgerungen ableiten, die hinsichtlich möglicher Steigerungen des Demokratieniveaus der Bevölkerungsmehrheit mit hoher Wahrscheinlichkeit weit über diejenigen beschränkten Erfolgsperspektiven hinausführen, zu denen man gelangt, wenn man sich etwa auf die Zukunftsperspektive einer gesellschaftsweiten Parteimitgliedschaft festlegt.

\section{Angela Merkel als Exempel? Ableitung möglicher Folgerungen}

Zusammengefasst beurteilt führt es kaum zu weit, wenn man angesichts der mitgeteilten Angela Merkel betreffenden Fakten von einem Exempel für die unter den gegenwärtigen Bedingungen bestehende Möglichkeit einer von einzelnen Personen maßgeblich mitgetragenen "personalen "Basis der Systemzuwendung der Bevölkerungsmehrbeit und damit einer elementar wichtigen Einstellungsgrundlage der ihr zuzurechnenden Demokratiequalität ausgeht.

Von hier aus gesehen legt sich eine Folgerung nahe, die zwar in der aktuellen Diskussionslandschaft der Bundesrepublik noch kaum verankert ist, die aber nichtsdestoweniger geeignet sein könnte, in dieser eine Schlüsselrolle zu spielen, sobald die ihr eigentlich zustehende Bedeutung angemessen gewürdigt wird. In allgemeiner Form ausgedrückt lautet diese Folgerung, dass das demokratische Potenzial, das der Bereitschaft der Bevölkerungsmehrheit zur Entwicklung einer personenbezogenen Systemzuwendung innewohnt, in Zukunft im Interesse der Stabilität und möglichsten Steigerung des gesellschaftlichen Demokratieniveaus wie auch der Qualität des demokratischen Gesamtsystems stärker als bisher genutzt werden sollte.

In unverschlüsselter Sprache ausgedrückt heißt dies erstens, dass bei die Auswahl der Inhaber/innen der politischen Spitzenposition (lies: der Bundeskanzler-Rolle) die gesellschaftsbezogenen interpersonalen Fähigkeiten und nicht die Durchsetzungsfähigkeit im inner- und zwischenparteilichen Konkurrenzkampf um Machtpositionen ausschlaggebend sein sollten. Zweitens heißt dies aber auch, dass das Verfabren zur Auswabl einer geeigneten Persönlichkeit so weitgehend wie möglich der Gesellschaft selbst anvertraut werden sollte.

Konkret gesagt bedeutet dies, eine künftige Direktwabl des/der Bundeskanzler/in ins Auge zu fassen. 


\section{Abschließende Überlegungen}

Es soll nicht die Aufgabe dieses Textes sein, diesen Vorschlag detailliert abzuhandeln. Auf einige Einzelheiten seiner möglichen Umsetzung wird nichtsdestoweniger einzugehen sein, damit seiner möglichen Abqualifizierung als eine nur »dahingeredete « Utopie vorgebeugt wird.

So legt es sich nahe, zu betonen, dass für eine Direktwahl des Bundeskanzlers bzw. der Bundeskanzlerin nicht unbedingt ein zusätzlicher Wahlakt vonnöten wäre. Allerdings würde es sich nahelegen, den interessierten politischen Parteien bis zu einem möglichst frühen Zeitpunkt vor dem Termin der jeweiligen Bundestagswahl die verbindliche Nominierung eines Kandidaten/einer Kandidatin, wie auch die Veröffentlichung eines Wahlprogramms abzufordern, an dessen Abfassung der/die Kandidat/in persönlich maßgeblich beteiligt werden sollte und das er/sie - mit Zustimmung der Partei - mit Überzeugung als »sein«/«ihr « Programm vorstellen und vertreten kann. In der vorhergehenden Zeit sollten die Kandidaten oder Kandidatinnen ihre Vorüberlegungen zu dem geplanten Wahlprogramm mit einer möglichst breiten Öffentlichkeit erörtern können, der Möglichkeiten zu einer aktiven Mitsprache eingeräumt werden sollten.

Diejenige Partei, die bei der Wahl die meisten Stimmen erhält, sollte aufgrund dieses Ergebnisses automatisch den/die vorher als Kandidat/in nominierte(n) Bundeskanzler/in stellen können, dessen/deren Wahl im Bundestag somit entfallen würde. Der betreffenden Partei sollte es ggf. freistehen, Koalitionen mit anderen Parteien zum Zweck der Mehrheitsbildung zu schließen. Es sollte hierbei allerdings nicht zur Verabschiedung eines von ihrem Wahlprogramm abweichenden »Koalitionsprogramms « kommen. Die Bereitschaft zur grundsätzlichen Mitvertretung dieses Programms sollte vielmehr eine Bedingung der Koalitionsbildung sein.

Es würde damit - jedenfalls im vorliegenden Zusammenhang - die gegenwärtig oft mehr oder weniger gedankenlos vorgenommene Sakrosanktstellung des »Kompromisses « entfallen, der in Wahrheit keineswegs immer eine Garantie realitätsnaher Demokratie ist, sondern vielmehr - jedenfalls bei dem bisherigen Verfahren der Koalitionsbildung - das Vertrauen der Wähler/innen rücksichtslos schädigt und damit der Entfremdung zwischen den politischen Eliten und der Bevölkerung Vorschub leistet (vgl. auch oben).

Die Erzielung einer Mehrheit der Bundestagsabgeordneten auf dem Weg der Koalitionsbildung sollte nicht die Voraussetzung der Regierungsbildung der Partei mit den meisten Stimmen sein. Diese sollte vielmehr die Möglichkeit der Bildung einer Minderheitsregierung wahrnehmen können. Bei der Gefahr des Scheiterns wichtiger Gesetzesvorhaben sollte sie - unter bestimmten, den Ausnahmecharakter garantierenden Bedingungen - die Möglichkeit haben, beim Bundespräsidenten die Zuerkennung einer »verliehenen« Mehrheit zu beantragen.

Eine vereinfachende und etablierten Demokratievorstellungen entgegenkommende Alternative hierzu wäre das definitive Verbot des »Fraktionszwangs«, der den Abgeordneten gegenwärtig noch den in höchstem Maße dubiosen Verzicht auf die ihnen im 
Grundgesetz ausdrücklich zugebilligte Gewissensfreiheit bei allen Entscheidungen abnötigt. Die Machtstellung der Fraktionsvorsitzenden sollte ggf. entsprechend reduziert werden. An die Stelle der Herstellung einheitlicher Fraktionsstrandpunkte sollten einfache Aussprachen bei nachfolgender Freistellung der Abstimmung treten.

Der Vermehrung von »Demokraten« in der Bevölkerung, wie letztlich sogar im Bundestag, wäre so oder so ein Dienst erwiesen. 